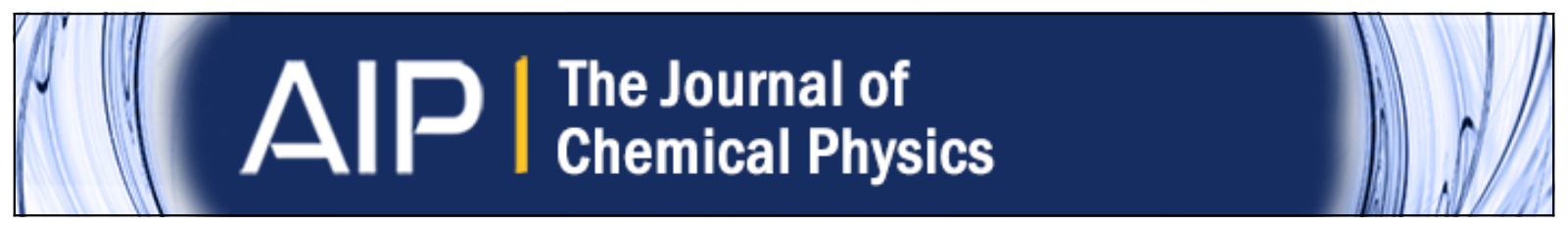

Mixed brush of chemically and physically adsorbed polymers under shear: Inverse transport of the physisorbed species

C. Pastorino and M. Müller

Citation: The Journal of Chemical Physics 140, 014901 (2014); doi: 10.1063/1.4851195

View online: http://dx.doi.org/10.1063/1.4851195

View Table of Contents: http://scitation.aip.org/content/aip/journal/jcp/140/1 ?ver=pdfcov

Published by the AIP Publishing

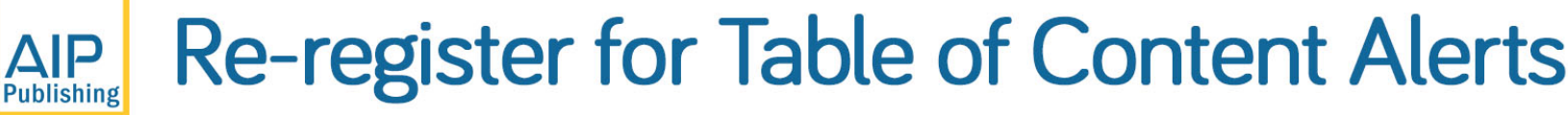




\title{
Mixed brush of chemically and physically adsorbed polymers under shear: Inverse transport of the physisorbed species
}

\author{
C. Pastorino ${ }^{1,2}$ and M. Müller ${ }^{2}$ \\ ${ }^{1}$ Departamento de Física de la Materia Condensada, Centro Atómico Constituyentes, CNEA/CONICET, \\ Av. Gral. Paz 1499, 1650 Pcia. de Buenos Aires, Argentina \\ ${ }^{2}$ Institut für Theoretische Physik, Georg-August Universität, Friedrich-Hund-Platz 1, 37077 Göttingen, \\ Germany
}

(Received 23 September 2013; accepted 5 December 2013; published online 6 January 2014)

\begin{abstract}
We study mixed brushes under shear flow by molecular dynamics simulation with an explicit solvent. The primary brush is formed by chemically grafting polymers to a solid substrate, the secondary brush is comprised of shorter, physically end-adsorbed molecules that can laterally diffuse. By virtue of the immobility of the grafted end-points of the primary brush, its individual macromolecules perform a cyclic motion. If there is a well defined solvent-brush interface, this cyclic motion of the primary brush molecules will collectively result in the reversal of the flow inside of the primary brush. This backflow, linear in the shear rate, gives rise to the transport of the shorter, physically end-adsorbed molecules in the opposite direction of the solvent flow. We discuss which conditions are necessary to observe this counter-intuitive phenomenon. Comparing Poiseuille and Couette flow we demonstrate that the magnitude of the local shear rate at the brush-liquid interface dictates the cyclic motion and concomitant inversion of transport but that these universal effects are independent of the type of driving the flow. @ 2014 AIP Publishing LLC. [http://dx.doi.org/10.1063/1.4851195]
\end{abstract}

\section{INTRODUCTION}

Polymer brushes have attracted abiding interest as versatile and stable functional surface coatings. By grafting long, flexible polymers to a substrate one can tailor adsorption and wetting properties as well as the fluid flow past these soft, synthetic, or biological surfaces. ${ }^{1}$ To a first approximation the brush can be conceived as a porous medium and Brinkman's equation ${ }^{2,3}$ has been employed to describe the flow inside the brush. Recent studies, however, reveal a more complex behavior: Simulations indicate strong modifications of the hydrodynamic boundary condition by brush coatings, which can be traced back to (i) an increase of the effective viscosity inside the brush ${ }^{4}$ and (ii) the cyclic motion of the grafted polymer chains in shear flow. Experiments ${ }^{5}$ observe that the flow reduction by a brush coating is unexpectedly larger than predicted by the simple poro-elastic model. One possible explanation for this experimental finding is the inversion of the total velocity field inside the brush that has been observed in simulations. ${ }^{6}$

The cyclic tumbling motion of individual long flexible chain molecules in shear flow has been observed by fluorescence microscopy experiments. ${ }^{7,8,29,30}$ This dynamics stems from a complex interplay between Brownian dynamics, chain relaxation, and hydrodynamics effects, as it is also the case for the cyclic dynamics of an isolated, grafted chain under flow. ${ }^{9-13}$ In a polymer brush, the collective behavior of tumbling grafted chains may result in the inversion of the total velocity profile in a particular zone, inside the brush. This has been observed in dynamic single-chain-in-meanfield simulations, ${ }^{6}$ unentangled-Lennard-Jones bead-spring models, ${ }^{6,14}$ as well as soft, coarse-grained dissipative particle dynamics (DPD) simulations. ${ }^{15}$
In this work, we exploit that the surface flow inversion, resulting from the collective but un-synchronized tumbling motion of the individual grafted chain molecules, can give rise to inverse macroscopic transport of physisorbed molecules against the solvent flow direction.

This inverse transport is observed for the first time and we demonstrate that the inverse transport velocity scales linearly with the shear rate of the main flow, allowing to compare our simulation results at rather high shear rates with smaller values of the shear rates in experiments.

Since the backflow velocity inside the primary brush is small, a stringent confinement of the secondary species is mandatory. Even a brief exposure of the secondary species to the strong main flow would overcompensate the inverse transport inside the primary brush. We meet this critical requirement by using a secondary physisorbed brush where one chain end is allowed to diffuse laterally but must not detach from the grafting surface. ${ }^{16}$

The reversal of surface transport can be an interesting new feature for microfluidic sorting strategies or process triggering and suggests a possibility for direct experimental verification of the collective motion of brushes under shear.

We employ linear Couette flow to study the different features of the effect. For a selected parameter set we compare these results to Poiseuille flow that mimics the pressure-driven flow routinely used in microfluidic devices. This quantitative comparison shows that the cyclic motion is rather independent of the type of (macroscopic) flow and that its magnitude is dictated by the shear rate at the brush-liquid interface.

In Sec. II we provide details of the molecular model and simulation techniques, and Sec. III presents the results of our molecular simulations. Our manuscript concludes with a 
discussion of possibilities for measuring the reported inverse flow and estimates for the expected orders of magnitude in experimental realizations.

\section{INTERACTION MODEL AND SIMULATION METHODS}

The primary brush is comprised of long, flexible Lennard-Jones bead-spring chains with $N_{b_{1}}=32$ beads that are irreversibly fixed with one end to a smooth, impenetrable substrate. The head beads are held fixed (irreversible grafting), by setting to zero the total force on them in each time step. The difference in molecular lengths gives rise to a viscosity contrast between polymer brush and monomeric solvent. The secondary physisorbed brush consists in shorter chains, $N_{b_{2}}=12$, with an end bead fixed in z-direction but free to diffuse along the surface. This physisorption is represented by setting to zero the total force of the heads in $\hat{z}$ direction $\left(f_{z}^{\text {head }} b_{2} \equiv 0\right)$ for each time step. In $x$ and $y$ the calculated force is retained allowing for the in-plane diffusion of the secondary chains.

The mixed polymer brush and the explicit, monomeric solvent are represented by Lennard-Jones particles. The general dependence of the potential is of the truncated and shifted form:

$$
U(r)=U_{\mathrm{LJ}}(r)-U_{\mathrm{LJ}}\left(r_{\mathrm{c}}\right),
$$

with

$$
U_{\mathrm{LJ}}(r)=4 \varepsilon\left[\left(\frac{\sigma}{r}\right)^{12}-\left(\frac{\sigma}{r}\right)^{6}\right],
$$

where $\epsilon$ represents the energy scale and $\sigma$ the excluded volume. Non-bonded interactions are cut-off at a distance $r_{\text {cut }}=\sqrt[6]{2} \sigma$.

The Lennard-Jones energy scale, $\epsilon$, is identical for all non-bonded interactions. The length scale of the LennardJones potentials between all like species is $\sigma$. The incompatibility between brush and solvent is represented by a slight non-additivity, $\sigma_{b_{1} l}=\sigma_{b_{2} l}=1.2 \sigma$.

Neighboring beads along the molecular backbone are connected by a finitely extensible nonlinear elastic potential. This widely studied potential for coarse-grained polymer models, ${ }^{17}$ takes the explicit form:

$$
U_{\mathrm{FENE}}=\left\{\begin{array}{ll}
-\frac{1}{2} k R_{0}^{2} \ln \left[1-\left(\frac{r}{R_{0}}\right)^{2}\right] & r \leq R_{0} \\
\infty & r>R_{0}
\end{array},\right.
$$

where the maximum allowed bond length is $R_{0}=1.5 \sigma$, the spring constant is $k=30 \epsilon / \sigma^{2}$, and $r=\left|\mathbf{r}_{\mathbf{i}}-\mathbf{r}_{\mathbf{j}}\right|$ denotes the distance between neighboring monomers.

All the beads interact with the wall through a 9-3 Lennard-Jones-like potential:

$$
V_{\text {wall }}(z)=\left|A_{w}\right|\left(\frac{\sigma_{w}}{z}\right)^{9}-A_{w}\left(\frac{\sigma_{w}}{z}\right)^{3} .
$$

The parameters were set to $A_{w}=2.0$ and $\sigma_{w}=1$. More details of the interaction model can be found in Ref. 14 .

We point out that the present binary brush significantly differs from previous studies ${ }^{6}$ because the brush is in a bad solvent and the solvent is of low molecular weight. The brush forms a dense melt-like layer, the chain conformations are only mildly stretched in contrast to a swollen brush. The reduced chain stretching facilitates height fluctuations of the free chain ends that are critical for flow inversion by the tumbling motion.

The simulation box has dimensions $26.25 \sigma \times 18.19 \sigma$ $\times 40 \sigma$, with upper and lower brush-coated walls (see Fig. 1). Periodic boundary conditions are applied in the two lateral directions parallel to the surface. The primary brush $\left(N_{b_{1}}=32\right)$ is composed of 100 chains per wall, while 40 physisorbed chains $\left(N_{b_{2}}=12\right)$ per wall formed the secondary brush. These values correspond to grafting densities $\rho_{b_{1}}=0.209 \sigma^{-3}$ and $\rho_{b_{2}}=0.084 \sigma^{-3}$ for the primary and secondary brush, respectively. The solvent comprises 6240 Lennard-Jones monomers. The combination of grafting density and molecular length of the primary brush and the nonadditivity between brush and solvent results in (i) a broad distribution of free ends of the grafted chains and (ii) a dry brush with a well-developed brush-solvent interface. The chains of the secondary brush are short enough for not reaching the brush-solvent interface.

We used molecular dynamics simulation in conjunction with the dissipative particle dynamics thermostat, ${ }^{18-20}$ which is Galilean invariant and takes into account hydrodynamic correlations, as shown previously for these coarse-grained models and physical conditions. ${ }^{21}$ Time is measured in units of $\tau=\sigma \sqrt{m / \epsilon}$ where $m$ is the mass of a bead. For the simulations, we take a time step $d t=0.002 \tau$. We impose linear shear (Couette) flow by moving the grafting surfaces of a slitlike channel at constant and opposite velocities $\pm v_{\mathrm{w}}$, resulting in a constant shear rate, $\dot{\gamma}=2 v_{\mathrm{w}} / D_{\mathrm{w}}^{*}$, at the middle of the channel, where $D_{\mathrm{w}}^{*}$ defines an effective channel width. ${ }^{22}$ We imposed also, for some case, Poiseuille flow by applying a constant body force $f_{x}$ to each bead of the liquid and the

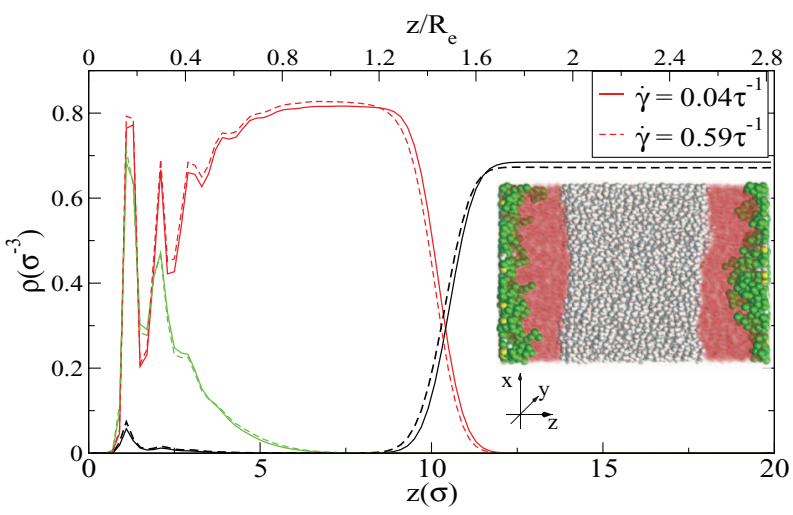

FIG. 1. Density profiles of the mixed brush in an explicit solvent for the lowest (solid lines) and highest (dashed lines) shear rates studied as indicated in the key in Lennard-Jones time units $\tau$. The grafted ends of the primary brush (red) are fixed to the surfaces that slide with constant velocities $\pm v_{\mathrm{w}}$ up and down. The physisorbed ends of the secondary brush (green) can move laterally. There is a well-defined interface between the primary brush (red) and the solvent (black), and the secondary brush (green) is confined between the grafting surface and the brush-solvent interface. The inset presents a configuration snapshot. The beads of the primary brush (red) are partially transparent to show the embedded secondary chains (green). The grafted and physisorbed ends of the primary and secondary brushes are indicated in light blue and yellow, respectively. 
primary brush. This gives rise to the usual parabolic velocity profile.

\section{RESULTS}

Fig. 1 presents the density profiles of all the species for the lowest and highest shear rates considered in this work. The height of the primary brush decreases only very slightly as we increase $\dot{\gamma}$ by about two orders of magnitude. A welldeveloped brush-solvent interface between the primary brush and the liquid solvent is formed with solvent particles remaining outside of the brush layer. The secondary brush is completely immersed in the primary one; only the largest fluctuations of its free ends get close to the brush-solvent interface as highlighted in the inset of Fig. 1. We also note that a small amount of solvent is trapped at the grafting surface, where one observes liquid-like layering effects (packing). The overall time-averaged structure perpendicular to the grafting surface and the position and width of the brush-solvent interface, in particular, are almost independent of the shear rate.

In the following, we measure velocity and length scales in terms of the Weissenberg number, $\mathrm{Wi} \equiv \dot{\gamma} \frac{R_{\mathrm{e}}^{2}}{3 \pi^{2} D}$, and the end-to-end distance $R_{\mathrm{e}}$ of the equivalent bulk polymer melt whose number density corresponds to that of the center of the primary brush, $\rho_{b 1}^{\text {bulk }}=0.82 \sigma^{-3}$. In this way, length and time are scaled with the typical spatial extent and relaxation time, $\tau^{*}=\frac{R_{e}^{2}}{3 \pi^{2} D}$, of a bulk polymer melt with the same molecular architecture and thermodynamic conditions as the primary brush layer. $\tau^{*}$ is the relaxation time of the longest Rouse mode of a polymer chain and $D$ is the self diffusion coefficient of a chain in a melt. ${ }^{23}$ We performed separate MD simulation for this bulk melt system with periodic boundary conditions in the three spatial directions and we obtained the values $R_{e}=7.06 \sigma, D=1.61 \times 10^{-3} \sigma^{2} / \tau$ and $\tau^{*}=1043 \tau$, respectively. From this values we can also estimate a stretching factor for the primary brush. We obtain a brush thickness $h_{\text {brush }} \simeq 10 \sigma$ (see Fig. 1) and therefore the stretching factor $S=h_{\text {brush }} / R_{e} \simeq 1.4$.

Fig. 2 shows the velocity profile for the three species. From the linear portion of the velocity profile of the solvent, we extract the shear rate $\dot{\gamma}$. The solvent velocity reaches the surface velocity, $v_{\mathrm{w}}$, in the vicinity of the brush-solvent interface. The time-averaged velocity of every bead of the immobile grafted primary brush coincides with $v_{\mathrm{w}}$. Nevertheless, there is a correlation between the fluctuating $z$-position of a primary brush bead and its lateral velocity that arises from the cyclic tumbling motion of the grafted macromolecules. The inset presents the velocity profiles in the frame that moves with the velocity of the surface at $z=0$. If a bead of the primary brush fluctuates away from the grafting surface to larger values of $z$, it is exposed to the greater solvent velocity at the brush-solvent interface, and it is dragged along with the flow. Therefore, there is a positive velocity (along the solvent flow) for the external part of the primary brush in contact with the solvent, $z / R_{\mathrm{e}} \simeq 1.3$. Since the overall velocity of a bead must average to zero, the bead tends to move in the opposite direction when it is closer to the grafting surface. Therefore, the

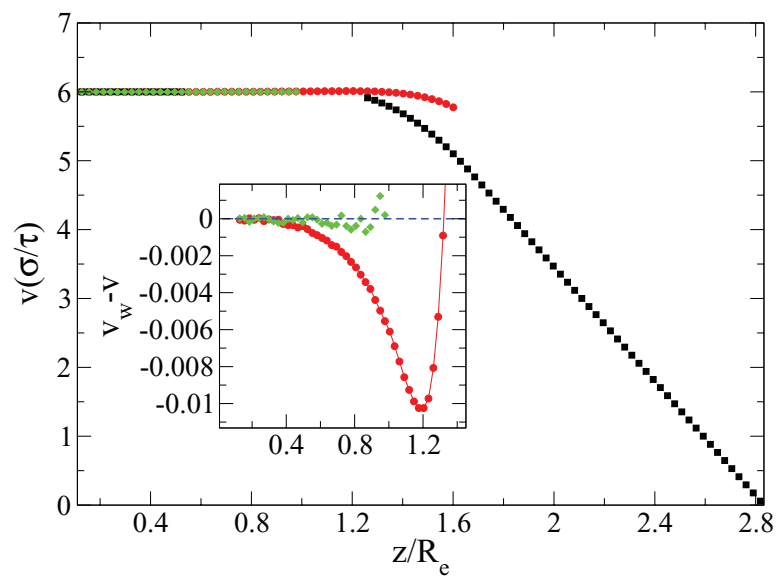

FIG. 2. Velocity profiles of both brushes (red and green) and the liquid solvent (black) for a shear rate of $\dot{\gamma}=0.59 \tau^{-1}$ or $\mathrm{Wi}=615.46 . z / R_{\mathrm{e}}=2.83 \sigma$ corresponds to the center of the channel. Data points, at which the local density is smaller than $1 \%$ of the bulk density, are omitted for clarity. Linear Couette flow is observed at the center of the slit-like channel. The (average) velocity of the brush beads agrees with that of the surface, $v_{\mathrm{w}}$, but the outmost beads of the primary brush are convected by the flow. Inset: Magnification of the velocity profiles $v^{\prime}=v_{w}-v$ for both brushes, close to the surface. The origin is set to wall velocity $\left(v^{\prime}=0\right)$ and the liquid flows in the positive $y$-axis direction. The collective cyclic motion of the primary brush (red) gives rise to a backflow inside the brush, which convects the secondary brush (green) against the direction of the solvent flow.

velocity of the primary brush beads is negative (against the solvent flow) in the interior of the brush.

In Fig. 3 we show the density profiles and mean velocity of end beads of the primary brush. The origin indicates the position of the grafted bead of the polymer chains. The profiles were obtained by averaging over all the grafted chains of both layers subjected to Poiseuille flow driven by an external body force $f_{x}=0.08 \epsilon / \sigma$. A maximum of the density of the end-bead is observed around $(x, z)=(17.5 \sigma, 9 \sigma)$ indicating the average tilting of the brush molecules. Additionally, in the outer part of the brush, the cyclic movement of the end-bead is clearly observed from the end-bead velocity field. Its shape and extension towards the interior of the brush determines the

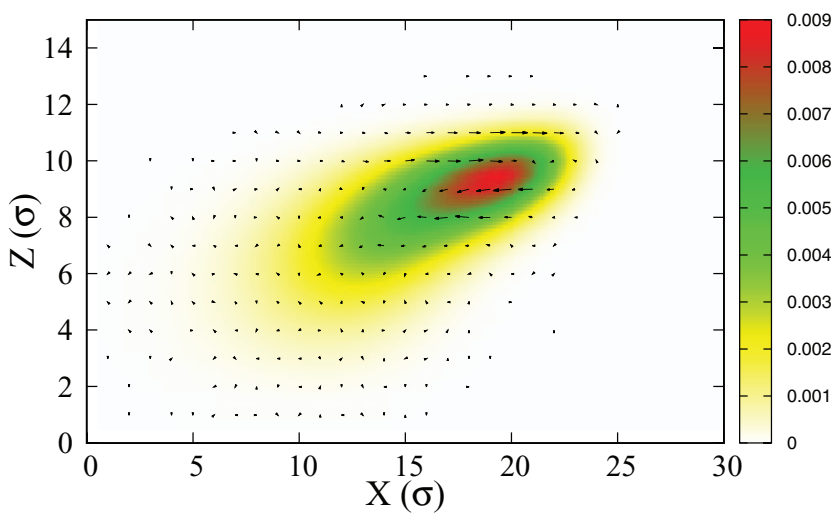

FIG. 3. Primary brush end-bead density (color plot) and velocity profiles. The cyclic dynamics and its spatial extension is clearly observed. The profiles were extracted from a Poiseuille flow simulation of body force $f_{x}=0.08 \epsilon / \sigma$. The axes are in units of $\sigma$. The vector plot is normalized with the maximum velocity set to one. The lower, left corner corresponds to the grafting point. 


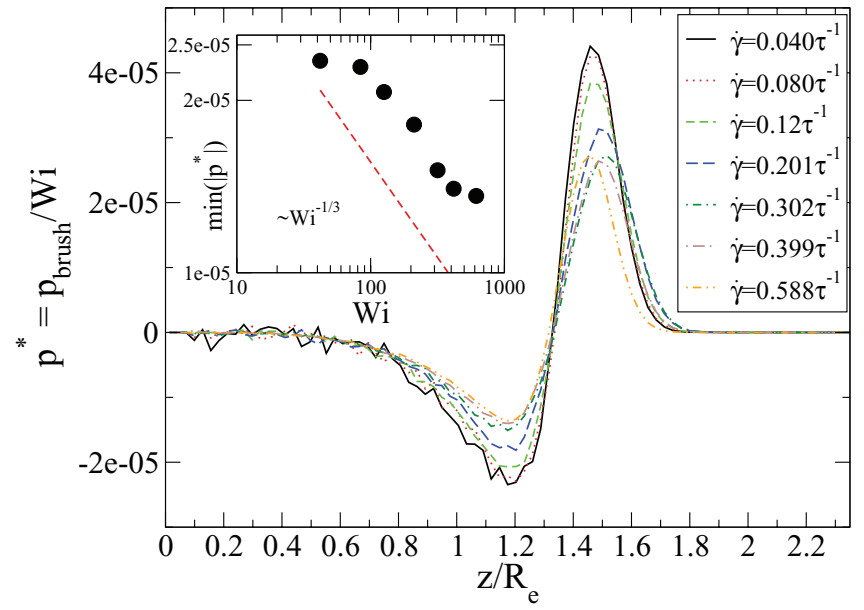

FIG. 4. Scaled momentum profiles $p^{*}=p / \mathrm{Wi}$ of the primary brush for different shear rates. The tumbling motion occurs in the region $0.8<z / R_{\mathrm{e}}<1.4$. The inset presents the spatial minimum of $p^{*}$ as a function of Weissenberg numbers. The dashed line corresponds to a scaling $\sim \mathrm{Wi}^{-1 / 3}$.

zone of inverted transport. Similar results have been obtained by self-consistent Brownian dynamics. ${ }^{24,25}$

In Fig. 4 we analyze the momentum density $p_{b 1}(z)$ $=\rho_{b 1}(z) v_{b 1}(z)$ of the primary brush as a function of the distance from the surface in the co-moving frame. There is no transport of the grafted primary brush and the integral of the momentum density profile vanishes in the co-moving frame. However, one clearly observes that the local momentum density is positive on the solvent-side of the brush-solvent interface and negative on the side towards the grafting surface.

In the figure, we have scaled the momentum density of the primary brush with the Weissenberg number, $p^{*}=p_{\text {brush }} / \mathrm{Wi}$, to rationalize the dependence on shear rate. For the lowest two shear rates, the scaled velocity profiles are independent from $\dot{\gamma}$, i.e., the velocity profile of the primary brush scales linear with the velocity of the solvent flow. This linear relation stems from the rotational component of the Couette velocity profile that couples to the motion of the grafted chains and results in their individual tumbling motion. At higher shear rates, $\mathrm{Wi} \gtrsim 60$, the velocity profile of the brush cannot completely follow the solvent. In this nonlinear regime, we observe that the minimal scaled momentum density, $\min \left(p^{*}\right)$, decreases like $\mathrm{Wi}^{-1 / 3},{ }^{26}$ i.e., the maximally inverted local momentum density scales with Wi to the power of $2 / 3$. This effective power law is indicated by a dashed line in the inset of Fig. 4.

Using extensive, multiple, independent molecular dynamics simulations of $8 \times 10^{7}$ time steps, we have investigated the motion of the secondary brush. The data for the velocity of the beads of the secondary brush as a function of their distance, $z$, from the adsorbing surface are shown in Fig. 2. Within the statistical uncertainty of the simulation data, there is no variation of the bead velocity with the distance $z$ but there is a small, constant negative velocity, i.e., the end-physisorbed molecules of the secondary brush are transported against the solvent flow because they are convected by the inverted flow generated by the tumbling motion of the primary brush.

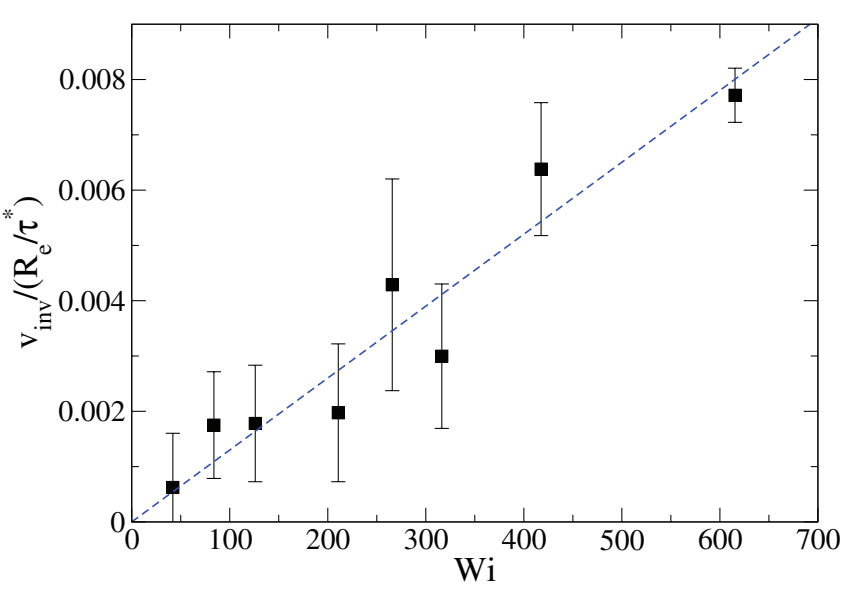

FIG. 5. Backflow velocity of the center of mass of the secondary brush as a function of Weissenberg number (scaled shear rate). The dashed line indicates that the velocity of the secondary brush against the solvent flow direction increases linearly with the shear rate.

In Fig. 5 we characterize this backflow by the center-ofmass velocity of the secondary brush as a function of the shear rate of the solvent. Within the accuracy of the simulation data, the backflow velocity is linearly proportional to the shear rate of the solvent flow. ${ }^{27}$

Quantitatively, the backflow velocity is small, but it is a qualitative effect. The convective nature of this motion implies that the backflow velocity $v_{\text {inv }}$ gives rise to a displacement $\left|\Delta x_{\text {inv }}\right| \sim v_{\text {inv }} t$, while the diffusive displacement scales as $\left|\Delta x_{\text {diff }}\right| \sim \sqrt{D_{2} t}$ with $D_{2}$ being the lateral selfdiffusion coefficient of the end-physisorbed species, i.e., for long enough times, backwards transport of the secondary chains will always dominate the diffusion.

A further question concerns the dependence of the inverse transport or the underlying cyclic motion on the type of flow. For computational efficiency, we have used Couette flow with a nearly constant shear rate in our simulations whereas pressure-driven flow with a linear shear-rate dependence is the more common way to impose flow in microfluidic devices. Using a pressure difference along the length of the channel gives rise to a quadratic velocity profile with a maximum at the center of the channel.

To study the effect of the macroscopic flow, we have performed Poiseuille-flow simulations by imposing an external body force, $f_{x}$ on each bead of the liquid and the primary brush, which also generates a parabolic mass-averaged velocity profile across the channel. The comparison of Couette and Poiseuille flow is presented in Fig. 6. The main panel shows the mass-averaged velocity profile for a Couetteflow simulation with shear rate $\dot{\gamma}=0.080 \tau^{-1}$ (dashed line) and a Poiseuille-flow simulation with external body force $f_{x}=0.02 \epsilon / \sigma$. These parameters are chosen such that the local shear rates at the brush-liquid interface is similar although the macroscopic flows are qualitatively different.

The inset of Fig. 6 displays the density profiles for primary brush and liquid, which are very similar. The slight difference of the apparent width of the brush-liquid interface can be partially traced back to the difference in the lateral system sizes and the broadening of the profiles due to local 


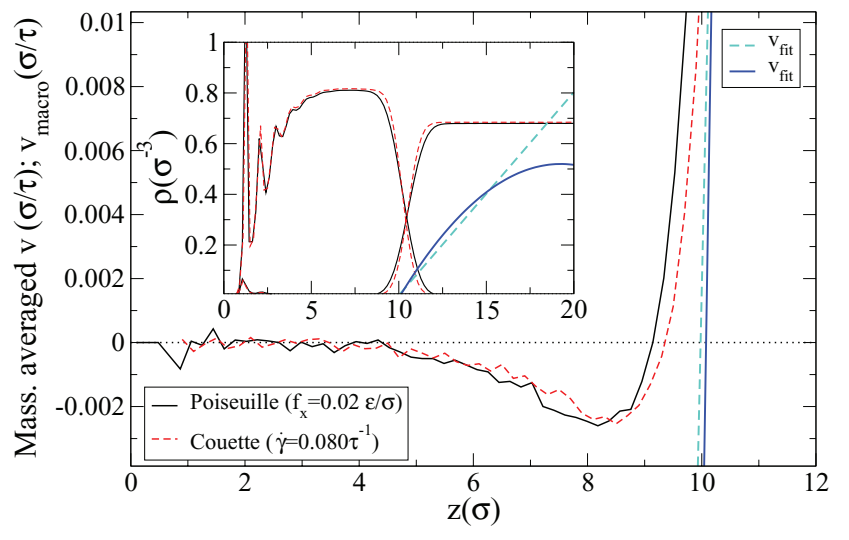

FIG. 6. Comparison of Couette-flow (dashed line, $\dot{\gamma}=0.08 \tau^{-1}$ ) and Poiseuille-flow (full line, $f_{x}=0.02 \epsilon / \tau$ ) giving rise to similar mass-averaged back-flow velocity profile of the primary brush. The fitted macroscopic velocity profiles, linear and parabolic, at the center of the channel are also presented. The inset shows the density profiles for both cases and the fitted macroscopic velocity profiles indicating the position of the hydrodynamic interface, $z \simeq 10 \sigma$.

fluctuations of the interface position. ${ }^{14}$ This panel also shows the extrapolated hydrodynamic velocity profiles that we obtained by fitting the macroscopic position dependence - linear for Couette flow and parabolic for Poiseuille flow, respectively - of the velocity profiles at the center of the channel. We observe that both types of flow extrapolate to zero velocity (i.e., the velocity of the grafting surface) at $z \simeq 10 \sigma$ indicating the location of the hydrodynamic boundary condition and vanishingly small slip.

Measuring local shear rates by fitting a linear curve in a small zone around $z \gtrsim 10 \sigma$ for the mass-averaged velocity profile, we obtain $\dot{\gamma}_{\text {loc }}=0.0521 \tau^{-1}$ and $\dot{\gamma}_{\text {loc }}=0.0522 \tau^{-1}$ for Poiseuille and Couette flows, respectively. The intensity of the cyclic motion of the brush and the concomitant inverse transport velocity are the same under these two qualitatively different macroscopic driving mechanisms demonstrating that both effects are determined by the local shear rate at the brushliquid interface. Therefore, we expect that the conclusions we draw from the Couette flow are robust, i.e., independent from the macroscopic flow away from the brush-liquid interface.

\section{DISCUSSION AND CONCLUSIONS}

One important condition for obtaining backwards transport is the presence of a well defined brush-solvent interface, in which the solvent does not penetrates in the brush layer (see Fig. 1). This requirement does not affect the cyclic tumbling motion of the irreversible grafted polymer chains per se but ensures that collective effect of the brush results in the inversion of the total momentum transport and that the effect is not wiped out by the solvent flow. Such a dry brush can be achieved by entropic effects, as in a brush-polymer melt interface, ${ }^{6,14}$ or by chemical incompatibility between the brush and the solvent molecules, as shown in this work. A minimal requirement for the existence of a brush-solvent interface is sufficient grafting density.

Another important pre-requisites are the thermal fluctuations of the position of the brush segments perpendicular to the wall because the tumbling motion relies on the correlation between height fluctuations and velocity. We anticipate that too large grafting densities, resulting in strongly stretched brush molecules with only very rare fluctuations of the free end segments to the high-velocity region of the solvent flow and to the vicinity of the substrate, or chain stiffness will decrease the tumbling motion and the concomitant backwards transport. Thus, we expect the backflow effect to occur at intermediate, experimentally accessible grafting densities, where the grafted chains overlap but are not strongly stretched.

We used a polymer brush in a bad solvent. Through the incompatibility between the brush and the solvent we can tailor the width of the brush-solvent interface and the molecular stretching of the grafted macromolecules remains small.

In order to estimate the possibility to observe the inverse transport experimentally, we map the coarse-grained model to realistic length and time scales using the observed relation between the velocity, $v_{\text {inv }}$, of the backflow, the extension of the primary brush $R_{\mathrm{e}}$, and the shear rate, $\dot{\gamma}$. From Fig. 5 we estimate $v_{\text {inv }}=\alpha R_{\mathrm{e}} \dot{\gamma}$ with $\alpha \approx 10^{-5}$. The detailed values of an experimental realization will strongly depend on the choice of the secondary brush. In our study we have chosen the secondary brush to have the same molecular architecture as the primary brush in order not to introduce further parameters. Using $R_{\mathrm{e}} \approx 33 \mathrm{~nm}$ for the primary brush (corresponding to a brush height of about $50 \mathrm{~nm}$ and an unperturbed end-to-end distance of $20 \mathrm{~nm}$ of the secondary brush) and a high shear rate of $10^{3} \mathrm{~s}^{-1}$, we estimate that the backflow velocity will be of the order of $v_{\text {inv }} \approx 3 \times 10^{-4} \mu \mathrm{m} / \mathrm{s}$. The crossover time scale from diffusive to convective transport is given by $t^{*}=D_{2} / v_{\text {inv }} \approx 100 \mathrm{~s}$ where we have used a diffusion constant of $10^{-5} \mu \mathrm{m}^{2} / \mathrm{s}$, prototypical for the bulk diffusion of a polystyrene chain with end-to-end distance $R_{\mathrm{e}, 2}=20 \mathrm{~nm} \cdot{ }^{28}$ Thus, backflow will dominate for distance larger than $v t^{*} \approx 0.03 \mu \mathrm{m}$.

This finding opens the possibility of developing a microfluidic device with brush-coated channels, having simultaneous transport in the direction of the solvent flow and, inside the brush, an opposite flow of a different molecule. Moreover, the backflow is highly independent of the flow type. Another set of simulations with a Poiseuille-type flow also resulted in backflow. We showed that the intensity of the cyclic motion is similar to that of the linear flow, providing that one gets similar shear rates at the brush melt interface. This Poiseuille flow mimics the "pressure-driven" flow typically used in microfluidics and we hope that our simulation study will be corroborated by experiments.

\section{ACKNOWLEDGMENTS}

Financial support by the SFB 937 "Collective behavior of soft and biological matter" TP A5, the German Academic Exchange Service (DAAD) as well as CONICET (PIP 08,11), MINCYT (PICT 11), and INN-CNEA grants is gratefully acknowledged. Computing time was also provided by the HLRN Hannover/Berlin and the JSC Jülich, Germany.

${ }^{1}$ K. Binder, T. Kreer, and A. Milchev, Soft Matter 7, 7159 (2011). 
${ }^{2}$ H. C. Brinkman, Appl. Sci. Res., Sect. A 1, 27 (1949).

${ }^{3}$ S. T. Milner, Macromolecules 24, 3704 (1991).

${ }^{4}$ D. I. Dimitrov, A. Milchev, and K. Binder, Macromol. Theory Simul. 17, 313 (2008).

${ }^{5}$ L. Lanotte, S. Guido, C. Misbah, P. Peyla, and L. Bureau, Langmuir 28, 13758 (2012).

${ }^{6}$ M. Müller and C. Pastorino, Europhys. Lett. 81, 28002 (2008).

${ }^{7}$ D. E. Smith, H. P. Babcock, and S. Chu, Science 283, 1724 (1999).

${ }^{8}$ S. Gerashchenko and V. Steinberg, Phys. Rev. Lett. 96, 038304 (2006).

${ }^{9}$ P. S. Doyle, B. Ladoux, and J. L. Viovy, Phys. Rev. Lett. 84, 4769 (2000).

${ }^{10}$ R. G. Winkler, Phys. Rev. Lett. 97, 128301 (2006).

${ }^{11}$ R. Delgado-Buscalioni, Phys. Rev. Lett. 96, 088303 (2006).

${ }^{12}$ C.-C. Huang, G. Sutmann, G. Gompper, and R. G. Winkler, Europhys. Lett. 93, 54004 (2011).

${ }^{13}$ Y. Zhang, A. Donev, T. Weisgraber, B. J. Alder, M. D. Graham, and J. J. de Pablo, J. Chem. Phys. 130, 234902 (2009).

${ }^{14}$ C. Pastorino, K. Binder, and M. Müller, Macromolecules 42, 401 (2009).

${ }^{15}$ M. Deng, X. Li, H. Liang, B. Caswell, and G. E. Karniadakis, J. Fluid Mech. 711, 192 (2012).

${ }^{16}$ We envision that molecules with a physisorbed group along the molecular contour will exhibit a similar behavior. This strategy may serve as a platform for inversely transporting other molecules that strongly bind to the physisorbed brush.

${ }^{17}$ G. S. Grest and K. Kremer, Phys. Rev. A 33, 3628 (1986).

${ }^{18}$ R. D. Groot and P. B. Warren, J. Chem. Phys. 107, 4423 (1997).

${ }^{19}$ P. Español and P. Warren, Europhys. Lett. 30, 191 (1995).
${ }^{20}$ T. Soddemann, G. K. Auernhammer, H. Guo, B. Dünweg, and K. Kremer, Eur. Phys. J. E 13, 141 (2004).

${ }^{21}$ C. Pastorino, T. Kreer, M. Müller, and K. Binder, Phys. Rev. E 76, 026706 (2007).

${ }^{22}$ C. Pastorino, K. Binder, T. Kreer, and M. Müller, J. Chem. Phys. 124, 064902 (2006)

${ }^{23}$ M. Doi and S. F. Edwards, The Theory of Polymer Dynamics (Oxford, 2001).

${ }^{24}$ M. G. Saphiannikova, V. A. Pryamitsyn, and T. Cosgrove, Macromolecules 31, 6662 (1998)

${ }^{25}$ M. G. Saphiannikova, V. A. Pryamitsyn, and T. M. Birshtein, Macromolecules 33, 2740 (2000).

${ }^{26}$ Experimental and simulation studies of individual, grafted molecules ${ }^{29,30}$ observed a $\mathrm{Wi}^{-1 / 3}$-scaling for the chain dimensions. We have verified, however, that the dependence of the molecular extension on the Weissenberg number in our brush is significantly weaker than for isolated chains. $R_{\mathrm{e}}$ varies only by a factor of 2 between $\mathrm{Wi}=0$ (equilibrium) and our highest shear rate, $\mathrm{Wi}=615$. We attribute this difference to the screening of the flow inside the brush

${ }^{27}$ Within the error bars the simulation data would also be compatible with a $\mathrm{Wi}^{2 / 3}$ for larger shear rates as suggested by the momentum profiles of the primary brush in Fig. 4.

${ }^{28}$ Note that the crossover time $t^{*}$ will be independent of the molecular weight in the regime where the diffusion of the secondary brush is Rouse-like and $D_{2} \sim \frac{1}{N_{2}}$ and $R_{\mathrm{e}, 2}^{2} \sim N_{2}$.

${ }^{29}$ B. Ladoux and P. S. Doyle, Europhys. Lett. 52, 511 (2000).

${ }^{30}$ S. Litvinov, X. Y. Hu, and N. A. Adams, J. Phys.: Condens. Matter 23, 184118 (2011) 